\title{
Second-Look Endoscopy After ESD: Can We Finally See the Forrest from the Trees?
}

\author{
Payal Saxena ${ }^{1,2,3,4}$
}

Published online: 20 August 2015

(c) Springer Science+Business Media New York 2015

Endoscopic mucosal resection (EMR)and endoscopic submucosal dissection (ESD) are frequently used in Asia as curative techniques for gastric adenoma and early gastric cancer (EGC) with low risk of lymph node metastasis. ESD is significantly more effective than EMR whether en bloc resection, complete resection, or curative resection is used, or in terms of local recurrence [1]. Nevertheless, ESD creates a large artificial ulcer that can be a source of intra- and post-procedural hemorrhage. Although intraprocedural bleeding can be controlled with hemostatic methods, post-procedural or delayed bleeding remains of significant concern. Delayed bleeding can manifest as hematemesis or melena within $24 \mathrm{~h}-1$ month after the procedure with reported rates as high as 5-38\% [2-4]. Therefore, prevention of delayed bleeding is a clinically important issue.

What can be done to prevent delayed bleeding following ESD? The submucosal vessels are undeniably injured during the procedure. Therefore, coagulating any visible vessels immediately post-procedure appears to be a reasonable strategy to prevent delayed bleeding. In a large retrospective study of 1083 gastric lesions, Takizawa et al. [2] reported reduced post-ESD bleeding rates in patients undergoing routine post-ESD coagulation (PEC; 7.1 vs.

Payal Saxena

psaxena1@jhmi.edu

1 Royal Prince Alfred Hospital, Camperdown, NSW 2050, Australia

2 Chris O'Brien Lifehouse, Camperdown, NSW 2050, Australia

3 Concord Hospital, Concord, NSW 2139, Australia

4 Division of Gastroenterology and Hepatology, Johns Hopkins Hospital, 1800 Orleans St, Baltimore, MD, USA
$3.1 \%, p<0.01$ ), a practice now employed at many centers. In order to further minimize delayed bleeding, secondlook endoscopy (SLE) after ESD is used to achieve hemostasis of high-risk lesions and is commonly performed. The Forrest classification (Table 1) is a valid tool for describing and predicting acute peptic ulcer lesions that are at high risk of re-bleeding. A recent meta-analysis of patients with acute peptic ulcer bleeding reported the effectiveness of SLE in reducing delayed bleeding (OR $0.55 ; 95 \%$ CI $0.37-0.81$ ) [5]. Nonetheless, the only included trial in which high-dose proton pump inhibitors (PPIs) were used did not support any benefit of SLE. Albeit, many ESD practitioners have extrapolated data from bleeding peptic ulcer cohorts to support the use of prophylactic hemostatic therapy during SLE based on the Forrest classification [6]. In particular, spurting/oozing vessels (Forrest Ia and Ib) and non-bleeding visible vessels (Forrest IIa and IIb) are treated with endoscopic coagulation or clipping. With routine PPI use, the morbidity and mortality of peptic ulcer disease have markedly decreased, de-emphasizing the utility and value of SLE in peptic ulcer bleeding. In the current PPI era, the value of routine SLE for ESD ulcers may also be controversial.

Several studies evaluating the effectiveness of SLE for ESD ulcers have actually been unfavorable. Two randomized controlled studies failed to support a beneficial effect of SLE in the prevention of delayed bleeding or the reduction in morbidity after $\operatorname{ESD}[7,8]$. Kim et al. reported no significant difference in the delayed bleeding rate between the SLE and non-SLE group (3.6 vs. $2.8 \%$, $p=0.79)$.

Regrettably, despite advanced and improved ESD techniques, the availability of accessories, and the use of prophylactic intra-operative hemostasis, the delayed bleeding rate is still not zero. Thus, many institutions 
Table 1 Forrest classification of peptic ulcers

\begin{tabular}{ll}
\hline Forrest classification & \\
\hline Ia & Active spurting vessel \\
$\mathrm{Ib}$ & Active oozing vessel \\
$\mathrm{IIa}$ & Non-bleeding visible vessel \\
$\mathrm{IIb}$ & Adherent clot \\
$\mathrm{IIc}$ & Flat pigmented spot \\
$\mathrm{III}$ & Clean base
\end{tabular}

continue to perform routine SLE after gastric ESD. Goto et al. [3] reported that $50 \%$ of patients who experienced delayed hemorrhage not only bled after SLE, but also did not have bleeding-risk stigmata identified during SLE.

These data beg the question: Are the right lesions treated during SLE? In this issue of Digestive Diseases and Sciences, $\mathrm{Na}$ and colleagues in a large prospective study evaluated 706 gastric ESD procedures [9]. In this singlearm study, the authors evaluated the rate of late delayed bleeding when non-bleeding visible vessels were identified (Forrest IIa) but not treated during SLE after ESD of gastric lesions, in comparison with Forrest IIb, IIc, and III lesions.

All patients received intravenous PPI from the morning of the procedure followed by an oral PPI for 4-8 weeks. PEC was performed in all cases. Complete blood cell counts and chest X-ray results were checked on the following day. An oral diet was begun if there was no evidence of bleeding and perforation. All patients underwent SLE on day 2 post-ESD. Yet, contrary to established practices, the authors only performed hemostatic therapy on Forrest Ia and Ib lesions. If Forrest IIa, IIb, IIc, or III lesions were identified at SLE, they were not treated. All patients were evaluated in an outpatient clinic 1 month after ESD with repeat hemoglobin concentrations measured.

Median specimen size was $40 \mathrm{~mm}$. At SLE, $8.9 \%$ of cases had Forrest I lesions that were treated without any further bleeding post-discharge. Strikingly, a majority $(82.5 \%)$ of these patients did not manifest clinical signs of upper gastrointestinal bleeding. Forrest IIa lesions were identified in $8.8 \%$, Forrest IIb in $16.9 \%$, and Forrest IIC/ III in $65.5 \%$ of cases. Overall, delayed bleeding occurred in $13.4 \%$ of cases, whereas late delayed bleeding (after SLE, within 1 month of ESD) occurred in $4.1 \%$ of cases; all were controlled with endoscopic methods (coagulation/clipping) without any need for surgical intervention.

Notably, the bleeding site did not correspond well to bleeding stigmata identified during SLE. Only two lesions with high-risk features such as an adherent clot or pigmented spot recognized at SLE were responsible for delayed bleeding. Fourteen cases occurred in other locations where no stigmata were observed during SLE. A further 11 cases could not be well defined as the bleeding was assessed at other hospitals.

Most significantly, there was no difference in the late delayed bleeding rate between Forrest IIa (3.2 \%), Forrest IIb $(4.2 \%)$, and Forrest IIc/III $(4.8 \%)$ lesions $(p=1.00)$. Larger specimen size was further confirmed as a risk factor for delayed bleeding. According to univariate analysis, resection size was the only factor that exhibited a trend toward prediction of late delayed bleeding ( $45 \mathrm{vs.} 40 \mathrm{~mm}$, $p=0.08$ ).

The authors must be commended for publishing the first prospective study that supports the hypothesis that prophylactic treatment of non-bleeding visible vessels does not influence delayed bleeding rates post-gastric ESD. Indeed, these results suggest that the Forrest classification cannot be applied to post-endoscopic resection ulcers for prediction of delayed bleeding. One of the reasons proposed by the authors lies in the major differences in the conditions surrounding ulcer formation when comparing post-ESD and peptic ulcers. Peptic ulcers are usually created in a low $\mathrm{pH}$ environment, whereas post-ESD ulcers are created under a relatively high $\mathrm{pH}$ due to the use of preand post-procedure PPI therapy [3]. The authors correctly postulated that ESD ulcers should heal faster due to the relatively alkaline environment, a concept highlighted in their methodology by withholding prophylactic treatment of non-bleeding visible vessels using SLE.

It is difficult to recommend cessation of SLE altogether since a substantial proportion of patients indeed had subclinical hemorrhage in this study. So perhaps the more important question to ask is not whether or not SLEs should be performed, but rather "who should undergo second-look endoscopy?"

Risk stratification is needed to identify the patients at greatest risk for delayed bleeding in order to develop criteria for SLE. In addition to a larger specimen size, procedure time, older age, high-grade histology, the use of antithrombotic drugs, the presence of submucosal fibrosis during ESD, and nausea are predictors of delayed bleeding $[8,10]$.

This study establishes a foundation for letting visible vessels rest as their prophylactic treatment contributes little to the prevention of late delayed bleeding. A prospective, randomized study of high-risk patients and lesions is warranted to ascertain the true yield and value of SLE after gastric ESD.

\section{References}

1. Park YM, Cho E, Kang HY, Kim JM. The effectiveness and safety of endoscopic submucosal dissection compared with endoscopic mucosal resection for early gastric cancer: a 
systematic review and metaanalysis Surg Endosc. 2011;25:26662677; Cao Y, Liao C, Tan A, Gao Y, Mo Z, Gao F. Meta-analysis of endoscopic submucosal dissection versus endoscopic mucosal resection for tumors of the gastrointestinal tract. Endoscopy. 2009;41:751-757.

2. Takizawa K, Oda I, Gotoda T, et al. Routine coagulation of visible vessels may prevent delayed bleeding after endoscopic submucosal dissection-an analysis of risk factors. Endoscopy. 2008;40:179-183.

3. Goto O, Fujishiro M, Kodashima S, et al. A second-look endoscopy after endoscopic submucosal dissection for gastric epithelial neoplasm may be unnecessary: a retrospective analysis of postendoscopic submucosal dissection bleeding. Gastrointestinal endoscopy. 2010;71:241-248.

4. Nakamura M, Nishikawa J, Hamabe K, et al. Risk factors for delayed bleeding from endoscopic submucosal dissection of gastric neoplasms. Scandinavian journal of gastroenterology. 2012;47:1108-1114.

5. El Ouali S, Barkun AN, Wyse J, et al. Is routine second-look endoscopy effective after endoscopic hemostasis in acute peptic ulcer bleeding? A meta-analysis Gastrointestinal endoscopy. 2012;76:283-292.

6. Chung IK, Lee JH, Lee SH, et al. Therapeutic outcomes in 1000 cases of endoscopic submucosal dissection for early gastric neoplasms: Korean ESD Study Group multicenter study Gastrointestinal endoscopy. 2009;69:1228-1235; Uedo N, Takeuchi Y, Yamada Tet al. Effect of a proton pump inhibitor or an $\mathrm{H} 2-$ receptor antagonist on prevention of bleeding from ulcer after endoscopic submucosal dissection of early gastric cancer: a prospective randomized controlled trial. The American journal of gastroenterology.. 2007;102:1610-1616.

7. Ryu HY, Kim JW, Kim HS, et al. Second-look endoscopy is not associated with better clinical outcomes after gastric endoscopic submucosal dissection: a prospective, randomized, clinical trial analyzed on an as-treated basis. Gastrointestinal endoscopy. 2013;78:285-294.

8. Kim JS, Chung MW, Chung CY, et al. The need for second-look endoscopy to prevent delayed bleeding after endoscopic submucosal dissection for gastric neoplasms: a prospective randomized trial. Gut and liver. 2014;8:480-486.

9. Na S, Ahn JY, Choi KD, et al. Delayed bleeding rate according to the forrest classification in second-look endoscopy after endoscopic submucosal dissection. Dig Dis Sci. (Epub ahead of print). doi:10.1007/s10620-015-3693-x.

10. Choi CW, Kim HW, Kang DH, et al. Clinical outcomes of second-look endoscopy after gastric endoscopic submucosal dissection: predictive factors with high risks of bleeding. Surgical endoscopy. 2014;28:2213-2220. 